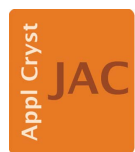

JOURNAL OF

APPLIED

CRYSTALLOGRAPHY

ISSN 1600-5767

Received 16 December 2017

Accepted 16 December 2017

Keywords: X-ray serial microcrystallography; sparse data; EMC algorithm; protein microcrystallography; synchrotron radiation sources

\section{Reconstructing three-dimensional protein crystal intensities from sparse unoriented two-axis X-ray diffraction patterns. Corrigendum}

\author{
Ti-Yen Lan, ${ }^{a}$ Jennifer L. Wierman, ${ }^{b, c}$ Mark W. Tate, ${ }^{a}$ Hugh T. Philipp, ${ }^{a}$ Veit Elser ${ }^{a}$ \\ and Sol M. Gruner ${ }^{a, b, c, d_{*}}$
}

\begin{abstract}
aLaboratory of Atomic and Solid State Physics, Cornell University, Ithaca, NY 14853, USA, bornell High Energy Synchrotron Source (CHESS), Cornell University, Ithaca, NY 14853, USA, 'Macromolecular Diffraction Facility at CHESS (MacCHESS), Cornell University, Ithaca, NY 14853, USA, and ${ }^{\mathbf{d} K a v l i}$ Institute for Nanoscale Science, Cornell University, Ithaca, NY 14853, USA. *Correspondence e-mail: smg26@cornell.edu
\end{abstract}

A figure in the article by Lan, Wierman, Tate, Philipp, Elser \& Gruner [J. Appl. Cryst. (2017), 50, 985-993] is corrected.

The color code of the curves in Fig. 3 on p. 989 of the article by Lan et al. (2017) is incorrect. The correct code (as shown in Fig. 1) is as follows:

Topmost (black) curve: local $\left(n_{\mathrm{c}}, n_{\mathrm{f}}\right)=(60,150)$

Second from top (blue) curve: local $\left(n_{\mathrm{c}}, n_{\mathrm{f}}\right)=(60,100)$

Third from top (red) curve: standard, $n=40$

Bottom (green) curve: local $\left(n_{\mathrm{c}}, n_{\mathrm{f}}\right)=(40,60)$

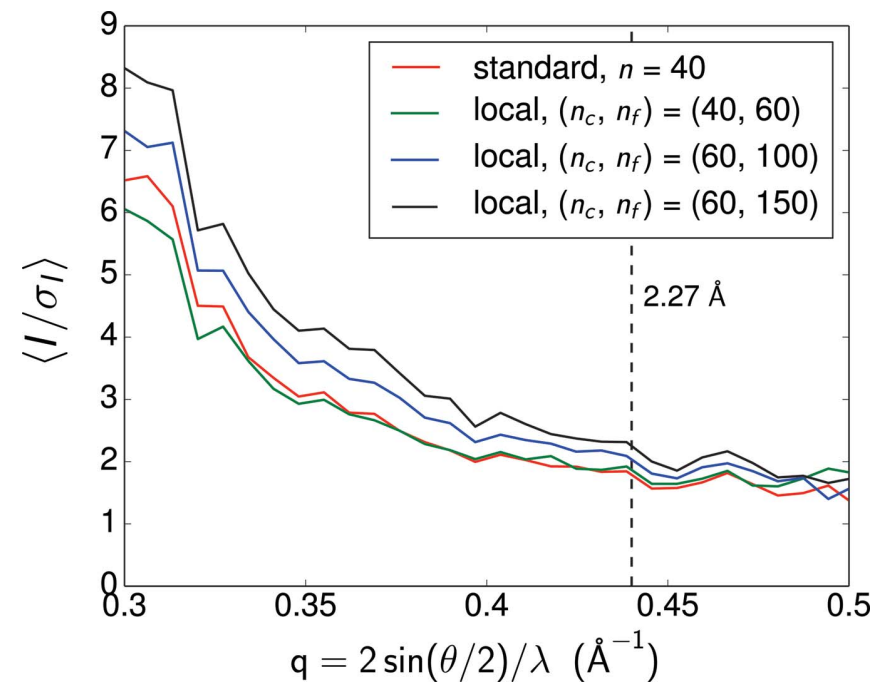

Figure 1

The corrected version of Fig. 3 of Lan et al. (2017). The average signal-tonoise ratio of the integrated reflections from the converged intensity maps at different stages of the reconstruction. The increase of $\left\langle I / \sigma_{I}\right\rangle$ at high $q$ indicates the reconstruction of high-resolution peaks. The $2.27 \AA$ resolution determined by $\mathrm{CC}^{*}$ is marked by the black dashed line.

\section{References}

Lan, T.-Y., Wierman, J. L., Tate, M. W., Philipp, H. T., Elser, V. \& Gruner, S. M. (2017). J. Appl. Cryst. 50, 985-993. 